Meta

Journal des traducteurs

Translators' Journal

\title{
Le discours sur la traduction juridique au Canada1
}

\section{Judith Lavoie}

Volume 47, numéro 2, juin 2002

Traduction et terminologie juridiques

URI : https://id.erudit.org/iderudit/008009ar

DOI : https://doi.org/10.7202/008009ar

Aller au sommaire du numéro

Éditeur(s)

Les Presses de l'Université de Montréal

ISSN

0026-0452 (imprimé)

1492-1421 (numérique)

Découvrir la revue

Citer cet article

Lavoie, J. (2002). Le discours sur la traduction juridique au Canada1. Meta, 47(2), 198-210. https://doi.org/10.7202/008009ar

\section{Résumé de l'article}

Le présent article propose une étude de la perception de la traduction législative par la communauté juridique à travers l'analyse des articles parus sur le sujet depuis les trente dernières années. Cette analyse montre que la traduction est souvent perçue comme un processus déformateur, entraînant du même coup une perception négative tant du résultat final (le texte traduit) que de la profession de traducteur. Discréditée d'abord pour les multiples calques qu'on retrouvait dans les lois fédérales traduites, la traduction l'est ensuite pour d'autres raisons : elle vaut moins que l'original (ne parle-t-on pas de simple traduction ?), elle est incomplète, elle fait intervenir la subjectivité du traducteur qui en vient à assumer le rôle du rédacteur-législateur. Les critiques portant sur le caractère servile des traductions des lois canadiennes avaient certes un fondement, toutefois, la mauvaise réputation que s'est faite la traduction ne semble pas avoir changé. De nos jours encore se trouvent des juristes pour qui traduire est un acte simple, qui ne requiert aucune créativité.
Ce document est protégé par la loi sur le droit d'auteur. L’utilisation des services d’Érudit (y compris la reproduction) est assujettie à sa politique d'utilisation que vous pouvez consulter en ligne.

https://apropos.erudit.org/fr/usagers/politique-dutilisation/ 


\title{
Le discours sur la traduction juridique au Canada'
}

\author{
JUDITH LAVOIE \\ Université de Montréal, Montréal, Canada
}

\begin{abstract}
RÉSUMÉ
Le présent article propose une étude de la perception de la traduction législative par la communauté juridique à travers l'analyse des articles parus sur le sujet depuis les trente dernières années. Cette analyse montre que la traduction est souvent perçue comme un processus déformateur, entraînant du même coup une perception négative tant du résultat final (le texte traduit) que de la profession de traducteur. Discréditée d'abord pour les multiples calques qu'on retrouvait dans les lois fédérales traduites, la traduction l'est ensuite pour d'autres raisons: elle vaut moins que l'original (ne parle-t-on pas de simple traduction?), elle est incomplète, elle fait intervenir la subjectivité du traducteur qui en vient à assumer le rôle du rédacteur-législateur. Les critiques portant sur le caractère servile des traductions des lois canadiennes avaient certes un fondement, toutefois, la mauvaise réputation que s'est faite la traduction ne semble pas avoir changé. De nos jours encore se trouvent des juristes pour qui traduire est un acte simple, qui ne requiert aucune créativité.
\end{abstract}

\begin{abstract}
This paper examines the jurists' perception of legislative translation through an analysis of the literature published over the last thirty years. The analysis shows that translation has long been seen as a process that, resulting in a negative perception of both the final product (the translated text) and distorts the translator's profession. At first stigmatized because of the numerous calques which appeared in the federal statutes translated into French; translation was then criticized for other reasons: it was considered to be a secondary or derivative activity (the words simple translation are often used to describe the process); it was also said to be incomplete; and it was seen as subjective given that the translator took up the role of the legislator. Criticism concerning the literalist tendency in translation was undoubtedly justified, but translation's bad reputation persists. Still, today, there are jurists for whom translating is a simple process which requires no creativity.
\end{abstract}

\section{MOTS-CLÉS/KEYWORDS}

traduction juridique, bilinguisme législatif, corédaction législative, interprétation législative

\section{Introduction}

Ancien juge à la Cour suprême du Canada, Louis-Philippe Pigeon écrivit un jour que, avant la promulgation de la Loi sur les langues officielles en 1969, les lois « [...] étaient publiées dans les deux langues, mais la rédaction en était unilingue et la traduction servile» (1982: 273). Pourrait-on, sans passer pour un véritable hérétique, nier le caractère littéral des traductions des lois fédérales au Canada? Pigeon avait bien sûr raison, les traductions nécessitaient sans conteste une nette amélioration, mais de quelle manière est-on passé de la critique du produit à celle de l'activité; comment et pourquoi la traduction fut-elle déconsidérée au sein du discours juridi- 
que? Une étude des articles parus sur le sujet depuis les trente dernières années montre en effet que la traduction est souvent perçue comme un processus déformateur, entraînant du même coup une perception négative tant du résultat final (le texte traduit) que de la profession de traducteur. Nous aurons donc pour tâche, dans un premier temps, d'énumérer les procédés par lesquels cette activité a été l'objet de critiques, et, dans un deuxième temps, de décrire la façon dont elle fut évincée du processus du bilinguisme législatif afin d'être remplacée par un mode de corédaction des lois fédérales. Discréditée d'abord pour les multiples calques dont étaient truffées les lois fédérales traduites, la traduction l'est ensuite pour d'autres raisons: elle vaut moins que l'original (ne parle-t-on pas de simple traduction?), elle est incomplète, imparfaite et fait intervenir la subjectivité du traducteur qui en vient à assumer le rôle du rédacteur-législateur... Mais avant d'aborder ce cortège de reproches, il convient de faire le point sur deux concepts fondamentaux dans le domaine de l'interprétation bilingue des lois fédérales: la règle d'égale autorité et le caractère critique du traduire.

\section{Les fondements théoriques du bilinguisme législatif}

Le principe selon lequel les deux versions d'un texte législatif ont force de loi, soit la règle d'égale autorité, représente sans aucun doute un concept théorique se situant au cour de l'interprétation législative bilingue. Selon l'article 133 de la Loi constitutionnelle de 1867: "Les lois du parlement du Canada et de la législature de Québec devront être imprimées et publiées dans ces deux langues [i.e. le français et l'anglais]». Contenue en germe dans la Constitution canadienne, la règle d'égale autorité sera consacrée en 1891 dans la décision C.P.R. c. Robinson, et en 1935 par la Cour suprême du Canada dans l'arrêt Dubois ${ }^{2}$.

Découlant en quelque sorte du premier, le second concept abordé postule que deux versions d'une loi peuvent servir conjointement à l'interprétation, qu'elles s'éclairent mutuellement donnant ainsi à l'interprète une meilleure compréhension et même une nouvelle saisie du sens. Résultant de l'égale autorité des deux versions, cette double mise à contribution des textes fait ressortir du même coup le caractère herméneutique du traduire, un postulat que plusieurs juristes mettent implicitement de l'avant. C'est le cas de John D. Honsberger, un avocat de Toronto, qui affirme, dès 1965, dans la Revue du Barreau canadien que: "Each version is of equal authority and [...] one may be used to interpret the other» (p. 327).

Roderick A. Macdonald écrit la même chose plus de trente ans après dans la Revue de droit de McGill: "One must supplement one version with the other and recognize that the text is incomplete without both. The presence of an equally authoritative set of propositions in two languages that must be reconciled can force an analysis of the spirit, intent and objects of an enactment - precisely those elements that are not explicit or formulaic» (1997: 144-145). Ce ne sont donc pas deux textes qui sont donnés à lire à l'interprète, mais, selon Macdonald, deux versions du même texte. Cette idée repose sur la conception selon laquelle l'intention législative n'est pas monopolisée par une seule langue, ni un seul texte, mais qu'elle est plutôt commune aux deux versions. Le corollaire, en droit, est que l'on doit considérer les deux textes comme des originaux lors de l'interprétation. Comme l'écrit André Labelle: "C'est donc dire que chaque version est un texte officiel, de valeur 
égale à celle de l'autre version, et qui peut précisément servir à l'interprétation de celle-ci» (2000: 271). Aucun texte n'est alors considéré comme une traduction. La traditionnelle scission entre original et version traduite est dès lors complètement escamotée par le biais de ce principe d'égalité des langues et des textes. Non pas seconde ni inférieure (deux défauts qui lui sont souvent imputés, comme nous le verrons plus loin), la traduction assume ici un rôle analogue à celui joué par le texte original, elle constitue un outil critique complémentaire aux méthodes traditionnelles d'interprétation législative. Bien qu'elle atteste du rôle primaire, et non accessoire, du traduire, la conception de Macdonald citée plus haut, ainsi que celle de Labelle, passent toutefois sous silence les mécanismes par lesquels le texte traduit constitue un réel instrument d'analyse du texte original. Ce sont précisément les différences, les écarts qui se creusent entre les deux versions d'un même texte qui donnent accès à des significations que la lecture simple aurait peut-être négligées. Or la traduction est généralement critiquée pour les mêmes raisons qui en font un procédé d'interprétation efficace : les déplacements inévitables qu'elle opère, les pertes qu'elle engendre, les gains qu'elle crée - ce que Barbara Folkart appelle la marge traductionnelle — constituent «[...] autant de lieux où le texte d'arrivée, non transparent par rapport au texte de départ, s'épaissit, acquiert une consistance propre, se constitue autre (il y a donc une altérité du texte d'arrivée, une altérité nécessaire et sur laquelle il faut insister, face au dogme de la fidélité). Les [...] artefacts créés par l'opération traductionnelle [...] définissent l'épaisseur de la traduction, une épaisseur faite des plus(ou des moins-) values produites par la ré-énonciation»(1991: 213). Ainsi, c'est dans l'hétérogénéité de la traduction que se fondent ses possibilités analytiques, la différence acquérant dès lors une valeur positive.

Pierre-André Côté, dans son ouvrage sur l'interprétation des lois, confirme le rôle herméneutique de la traduction considérant que le caractère bilingue des lois est utile à l'interprète puisque, «[...] bien souvent, le sens qui se dégage d'une version sera confirmé par la lecture de l'autre» (1999: 409).

Dans un article examinant le rôle assumé par la langue dans la définition du droit, Nicholas Kasirer, après avoir interrogé le lien étroit existant entre le français et la tradition de droit civil, demande si ce lien rend impossible l'expression du droit civil en anglais. Répondant par la négative, l'auteur insiste sur le fait que la langue anglaise recèle des ressources multiples et diverses pour exprimer les concepts civilistes. Ainsi, loin de voir, contrairement à plusieurs auteurs, le contact des langues comme un péril pour le droit, Kasirer constate qu'il entraîne un échange fécond dont chaque ordre juridique peut tirer parti: «[...] il existe un rapport de dépendance entre les deux versions linguistiques. Il y a, en effet, entre les textes législatifs français et anglais du droit privé québécois, une forme de dialogue » ${ }^{3}$ (1994: 162).

Jean-Claude Gémar, dont les travaux ont porté sur les questions de langue et de traduction juridiques au Canada, considère également que l'hybridité du droit, non seulement ne constitue pas une atteinte à l'intégrité de l'un ou l'autre des systèmes ou des langues en présence, mais peut avoir des retombées tout à fait bénéfiques:

La rencontre fortuite de deux «esprits des lois» opposés devait provoquer de toute évidence des effets inattendus, notamment dans l'activité traduisante, où le passage d'un système juridique à un autre présente des difficultés singulières. Or, au Canada, non seulement la plupart de ces difficultés, semble-t-il, ont été surmontées, mais il apparaît en outre que la traduction en général - en particulier de l'anglais au français 
et dans la province de Québec — a joué, au fil des décennies, un rôle unique de révélateur, provoquant un regain de la langue française qui a conduit à la revalorisation de son statut, par opposition à la langue de contact: l'anglais. (1995: XIV)

Enfin, l'étude de Rémi Michael Beaupré sur les règles d'interprétation bilingue fait état d'une multitude d'applications concrètes du principe d'égale autorité. En conclusion de son ouvrage, l'auteur note que:

Si l'on doit éviter le dogmatisme en parlant du droit qui semble rarement fixé sans être entouré de replis nombreux et complexes, notre examen de la jurisprudence a amplement démontré que la seule méthode d'interprétation digne de confiance de la législation bilingue au Canada exige, comme étape initiale, une lecture comparative des deux versions officielles.

Une telle conclusion découle de notre observation à partir d'innombrables décisions judiciaires que, fondée sur la règle d'égale autorité entre les versions française et anglaise, une version précise de la loi dissipera normalement tout doute entachant une version ambiguë et que le contexte d'une disposition résoudra normalement toute différence entre les deux versions. (1986: 189)

Une confirmation récente de ce principe a été donnée par la Cour suprême du Canada dans l'arrêt Doré c. Verdun ([1997] 2 R.C.S. 862). L'article 2930 du Code civil $d u$ Québec présentait deux interprétations opposées concernant la préséance des lois particulières dans l'application du délai de prescription en matière de préjudice corporel. Bien que la version anglaise du Code ait été qualifiée de simple traduction par le juge Baudoin de la Cour d'appel, la Cour suprême admettait qu'elle ne pouvait en faire fi compte tenu de la règle d'égale autorité des deux versions dans l'interprétation de la législation bilingue.

Malgré ces postulats d'égale autorité tant en droit qu'en fait, la traduction semble avoir été l'objet de critiques constantes au sein du champ juridique, critiques qui ont touché aussi bien sa nature transformatrice que la profession de traducteur, pour culminer dans le remplacement de l'activité elle-même par un nouveau mode de bilinguisme législatif: la corédaction.

\section{La traduction : opération transformatrice}

Le caractère foncièrement transformateur du traduire, qui est surtout perçu comme déformateur, a donné lieu à de nombreuses épithètes négatives de la part des auteurs — juristes, jurilinguistes, traducteurs — qui se sont penchés sur la traduction dans le domaine du droit.

Honsberger, dont les propos furent cités plus haut où il affirmait l'égale autorité des deux versions, considère que les versions françaises des lois fédérales sont trop littérales, qu'elles sont de mauvaise qualité et truffées d'anglicismes (1965: 336). Quatre ans plus tard, en 1969, Frederick Sussmann, un professeur de droit de l'Université d'Ottawa, lui emboîte le pas et écrit lui aussi que les traductions françaises sont trop littérales, ce qui entraîne, note-t-il: «[...] much clumsy and un-French sentence structure and practically meaningless, sometimes misleading French renditions of English technical words or expressions» (p. 19). À la suite de Pigeon, qui reprochait lui aussi aux traductions leur caractère servile (1982: 273), J.A. Clarence Smith, ancien professeur de droit de l'Université d'Ottawa, souligne le littéralisme des versions françaises et affirme que les traducteurs des lois fédérales étaient « [...] liés au 
texte anglais comme une ombre au corps qui la projette» (1983: 598). Macdonald, pour sa part, considère qu'en droit on est en présence de «mediocre translations » (1997: 156).

La qualité douteuse des textes traduits comporte certes un fondement, les mille et un calques dénoncés par les juristes ne furent pas inventés de toutes pièces, qu'on pense par exemple à la traduction du terme air gun par fusil à vent, au lieu de fusil à air comprimét, ou à celle de latent defaults par défauts latents au lieu de vices cachés ${ }^{5}$. Mais la recension de toutes ces erreurs ne fait pas de la traduction un procédé à proscrire. Car en plus d'être qualifiée de servile et donc de mauvaise qualité, la traduction est souvent considérée, de façon implicite ou explicite, comme un pis-aller, un mal nécessaire ou encore comme un sous-produit du texte original. Cette conception négative de la traduction — et son corollaire, la valorisation du texte original proviennent de la servilité non pas à l'auteur, mais au parlement. En effet, comme l'explique Kasirer, dans la théorie des sources du droit, l'idée selon laquelle le droit est totalement exprimé par le texte découle des positivistes: «[...] the conventional approach to interpretation [...] considered the role of the person charged with understanding meaning as less important, in service of the positivist ideal that the interpreter was not a creative agent in the theory of sources of law» (2001:334). Le positivisme fait donc en sorte que le juriste voit l'œuvre de traduction comme secondaire par rapport à celle du législateur.

Dénonçant le principe selon lequel il n'est pas opportun, pour des raisons d'égalité juridique, de dévoiler lequel des deux textes est la version traduite, Beaupré affirme qu'il serait utile de le faire afin d'être en mesure, à certaines occasions, d'écarter la traduction du processus interprétatif: «[...] l'article 8 [de la Loi sur les langues officielles, aujourd'hui abrogé] semble empêcher le développement de toute règle pragmatique d'interprétation qui permettrait l'établissement d'une hiérarchie entre les deux versions, hiérarchie fondée sur une indication ou une présomption que l'une des deux n'est qu'une simple traduction. Au cours des chapitres précédents, nous avons analysé un certain nombre de cas dans lesquels il était évident qu'une version était non seulement une traduction mais en plus une mauvaise traduction » (1986: 200, nos italiques). L'auteur présuppose ici que la traduction est un texte inférieur au texte original, un texte sans pouvoir, d'où l'avantage pour l'interprète du droit d'identifier entre les deux versions la véritable traduction. Le juge Baudouin de la Cour d'appel dans l'affaire Doré ne disait pas autre chose, qualifiant la version anglaise de «[...] simple traduction de la version originale française. Or, comme le dit si bien le proverbe italien «traduttore, traditore» (le traducteur est un traître)» (Cour d'appel [1995] R.J.Q., p. 1327). Bien qu'il s'agisse de traduction littéraire, les propos d'Antoine Berman s'appliquent à notre objet: «[...] le texte traduit paraît affecté d'une tare originaire, sa secondarité. Cette très ancienne accusation, n'être pas l'original, et être moins que l'original (on passe aisément d'une affirmation à l'autre), a été la plaie de la psychè traductive et la source de toutes ses culpabilités: ce labeur défectueux serait une faute (il ne faut pas traduire les œuvres, elles ne le désirent pas) et une impossibilité (on ne peut pas les traduire)» (1995: 42).

Emmanuel Didier, dans un ouvrage consacré à la jurilinguistique, définit les défauts de la traduction en ces termes: "La traduction n'est jamais complète, car le message transmis possède une structure que le message obtenu ne peut reproduire en totalité. [...] De plus, la traduction n'est pas totale, car, comme le langage ordi- 
naire, elle est impuissante à transférer toutes les connotations culturelles que le groupe ou l'individu attache au mot et à l'énoncé» (1990: 246). Sont ici dénoncées les lacunes inhérentes à tout acte de traduction et, à la limite, comme le fait remarquer l'auteur, à tout acte de communication. Incomplète, la traduction l'est toujours. Comme le constate Annie Brisset: "La traduction est un lieu par excellence d'empêchements et de tensions. Par nature, elle crée de la différence» (1990: 29). Il est effectivement impossible de rendre tous les référents culturels, idéologiques, politiques, sociaux contenus dans le message initial. La communication possède les mêmes limites: le destinataire du message n'est jamais à même de percevoir l'ensemble des visées originales de l'auteur et lui en attribue certaines que l'auteur n'avait ni souhaitées ni imaginées de façon consciente ou délibérée. Mais, pour Didier, et malgré cet aveu sur les lacunes du langage ordinaire (la loi s'apparente-t-elle au langage ordinaire?), la traduction demeure un procédé dont le caractère foncièrement approximatif et défectueux est toléré grâce à cette fiction juridique que constitue la règle d'égale autorité: «Le problème de l'imperfection de la traduction par rapport à l'original est aussi résolu sur le plan juridique par l'égalité des deux versions et l'obligation pour l'interprète de les consulter toutes les deux. Cette obligation est contraire à l'usage normal en matière de traduction, où l'on sait que l'original vaut toujours mieux que la traduction et où le lecteur n'est pas obligé de se baser sur la traduction » (1990: 248, nos italiques). Tablant sur un préjugé répandu au sein de la communauté juridique - la formulation rhétorique l'on sait que est utilisée - , Didier affirme que la traduction est sans conteste un texte inférieur à l'original. Cette présomption, en plus d'être issue du lien qu'établissent les juristes entre l'intention du législateur et le texte, repose sur le principe voulant que le texte de départ soit investi d'un caractère sacré, ce qui entraîne, du même coup, une idéalisation de l'original. Comme l'explique Kasirer, «[...] translators approach legal text as if it were a biblical text, bearing a single sacred message revealed to the reader by a close, literalist transcription into the target language» (2001: 340). Didier succombe à cette sacralisation du texte de loi: "La langue des lois n'est pas la langue courante: le souci de clarté et de précision du législateur lui fait choisir les mots nobles plutôt que les termes communs» (1990: 399). Dans ces conditions, il est presque naturel de croire au statut supérieur du texte source. Alexandre Covacs, traducteur, réviseur et jurilinguiste au ministère de la Justice du Canada en 1982, affirme d'ailleurs qu'avant les années 1980, c'est-à-dire avant l'instauration de la corédaction, «[...] le texte de départ était immuable par rapport au texte d'arrivée» (1982: 83). Seconde parce que résultant d'un texte au caractère immuable, la traduction ne peut être considérée autrement que comme un modeste sous-produit. Telle semble être l'opinion de Pierre-André Côté, qui écrit au sujet des lois bilingues que «[...] l'une des deux versions n'est que la traduction de l'autre... [qu'elle n'est que le] pâle reflet de l'autre version [...]» (1999: 418, nos italiques). Ainsi, pour Côté, traduire n'est pas faire œuvre de création, mais correspond plutôt à un travail de transposition. Cette affirmation, en plus de réitérer la prétendue secondarité de la traduction, présuppose qu'elle équivaut à un non-travail. Or, comme l'écrit Folkart:

[...] la traduction n'est jamais une transformation nulle. Elle ajoute toujours de la valeur, crée inévitablement des distorsions (bruit), des décalages ou des sollicitations (modulation) qui permettent de toucher du doigt à la ré-énonciation, de mesurer le décalage énonciatif qui se creuse entre la voix de l'instance de ré-énonciation et celle de 
l'instance d'énonciation, d'appréhender le dire du traducteur en flagrant délit de conflit avec celui de l'auteur.

De ce conflit d'énonciations témoignent les indices de ré-énonciation dont est entaché tout système traductionnel [...]. (1991: 127)

Paradoxalement, la traduction juridique est apparentée, ou bien à un sous-texte, dont l'effet principal est de dénaturer l'original, ou bien à une simple transposition, sorte de transvasement du sens d'un contenant linguistique à l'autre, mais dont le produit cible ne conserve ni l'éclat ni la richesse de la source.

Ainsi, il était presque dans l'ordre normal des choses que la traduction, après avoir récolté autant de reproches, soit écartée de la mise en application du bilinguisme législatif et remplacée par la corédaction. En fait, la traduction ne fut pas mise à l'écart de façon délibérée 6 , on a plutôt tenté de mettre au point des procédés qui permettraient d'éviter les multiples défauts découlant du processus traductionnel; la corédaction devait contrecarrer tous ces écueils.

\section{Traduire ou corédiger : des effets similaires?}

En 1978, après des décennies de traduction des lois de l'anglais vers le français, le ministère de la Justice du Canada met en œuvre un mode de corédaction: deux rédacteurs, un juriste anglophone et un juriste francophone, rédigent un texte de loi séparément et une équipe de jurilinguistes les conseille pour assurer une concordance entre les versions (Levert, 2000: 129). Mis à part le «court-circuitage» de la traduction qui résulte de l'instauration de cette méthode, ses principaux tenants affirment qu'elle aurait l'avantage, d'une part, d'éviter les interférences entre les deux langues, et d'autre part, de contrer l'influence de la structure de la phrase anglaise et de sa terminologie sur le français.

Mais ne serait-il pas juste d'affirmer que les résultats de la corédaction pourraient être atteints en traduction également? L'interférence entre les deux langues et l'influence de l'anglais sur le français ne sont-ils pas de faux arguments? En effet, la traduction, malgré des croyances tenaces, ne correspond pas à la simple reproduction, dans une autre langue, du texte original; nul n'est obligé de suivre la structure de la phrase anglaise à la trace, ni d'emprunter des termes à la langue source. J.A. Clarence Smith, pour sa part, admet que la traduction de qualité existe: "Le "littéraliste" sera peut-être choqué de trouver que la traduction [en Ontario] laisse froidement tomber, dans la mesure du possible, de multiples mentions du texte anglais qui, du point de vue français, sont inutiles. À titre d'exemple élémentaire, au lieu de répéter trois fois dans trois lignes "le Commissaire", on ne conserve qu'une fois le substantif, pour y substituer ensuite le pronom personnel» (1983: 599-600).

Autre avantage de la corédaction, qui pourrait au demeurant être présent dans un processus de traduction, la possibilité de corriger les deux textes, et non plus seulement le texte traduit. C'est ce qui a la faveur de Gérard Bertrand, premier conseiller législatif du gouvernement canadien, qui affirmait dans une conférence au congrès annuel de l'Institut international du droit d'expression française (IDEF) : «De surcroît, le texte de départ n'est plus, contrairement à ce qui se passe en traduction "classique", immuable, car le corédacteur en second peut amener le premier responsable du projet à modifier celui-ci et, ainsi, à l'améliorer» (1987: 5). Cette concertation entre les deux corédacteurs pourrait aussi se réaliser entre le traducteur et le rédacteur. C'est 
ce que proposait Normand Bélair en 1987, alors collaborateur à la traduction des lois de l'Ontario: "La traduction est la méthode qui s'impose naturellement quand il s'agit de transposer dans une autre langue des textes existants. [...] si le travail est fait consciencieusement, le traducteur signale au conseiller législatif les difficultés que pose l'original dans un contexte de bilinguisme. Les notes du traducteur devraient normalement avoir une incidence sur le texte de départ qui, avec le temps, s'adapterait ainsi à certaines exigences de la langue d'arrivée tant sur le plan de la perception de la réalité juridique que sur celui de son expression» (p. 6). Le caractère quasi sacré et intouchable du texte de départ serait de la sorte mis en question; la traduction, quant à elle, pourrait y gagner une certaine reconnaissance.

Macdonald, quant à lui, encourage la corédaction parce qu'il considère qu'elle permet de respecter le caractère idiomatique de chaque langue et de chaque culture juridique. Il soutient par exemple que, dans le cas d'une loi corédigée, chaque version pourrait ne pas comporter nécessairement le même nombre d'articles; il propose aussi que la version française pourrait contenir moins d'énumérations que la version anglaise, qui est plus portée à énumérer tous les cas de figure que le français, plus synthétique (Macdonald, 1997: 160). Or ces objectifs pourraient être atteints par le biais de la traduction également; une concordance parfaite entre les textes ne devrait pas être obligatoire. Ce fut pourtant le cas pendant de nombreuses années au ministère de la Justice fédéral. Macdonald n'a donc pas tort d'affirmer que: "Any attempt to achieve a bilingual statute-book through the translation of legislation initially drafted in one language into another cannot fully succeed. The inevitable limits of discursivity are such that translators will be compelled to sacrifice meaning for textual exactitude, and this sometimes even at the expense of clarity» (1997: 159). L'auteur entend par textual exactitude, la correspondance matérielle entre les deux versions. Une telle contrainte ne pouvait qu'entraîner des résultats décevants.

Covacs est lui aussi un défenseur de la corédaction, mais il pousse le raisonnement un peu plus loin: "Par un radical renversement de perspective, l'accent est d'entrée de jeu mis, non plus sur la traduction, mais sur la rédaction. Le français, trop souvent considéré un peu partout au Canada, au moins pour l'écrit, comme langue de traduction, se trouve du coup revalorisé en tant que langue du droit au même titre que l'anglais» (1982: 86, nos italiques). Ainsi, lorsqu'elle sert à traduire, une langue perd son autonomie, n'étant qu'à la remorque de la langue source.

En fait, l'instauration de la corédaction reposait sur une volonté de réparer les inégalités du passé. En effet, il s'agissait davantage de respecter la culture juridique et linguistique française du Canada que de pallier la mauvaise qualité des versions traduites en français. Dans la foulée du respect des deux langues officielles au Canada, on a décidé de changer les anciennes pratiques afin de donner au juriste francophone un statut égal au juriste anglophone. Un tel projet était donc sous-tendu par des enjeux politiques et non seulement par des préoccupations matérielles. On peut aussi supposer que l'arrivée au pouvoir du Parti québécois en 1976, lequel faisait de la promotion du français une mesure importante, a joué un rôle non négligeable dans la mise sur pied de la méthode de corédaction, Gémar affirmant d'ailleurs que: "C'est au cours de cette période que s'opère le passage d'une société de traduction à une société d'expression. Le pas décisif sera franchi en 1977, avec la Charte de la langue française, qui ne représente plus un simple projet de loi, mais bien un projet de société» (2000: 253). Ainsi, l'avènement de la corédaction visait également une 
valorisation de la contribution des francophones à l'ensemble du processus législatif. Keith Spicer, commissaire aux langues officielles, écrit dans son rapport, en 1977: "C'est dans le domaine de la législation qu'ont été relevées les entraves les plus fondamentales à l'égalité de statut des langues officielles. En dépit d'une amélioration notable de la version française des lois, il reste que, les projets de loi étant rédigés en anglais au départ, leur version française demeure, de l'avis général, un calque de l'approche de la common law alors que le texte français devrait refléter le génie de la langue française tout en englobant les principes des deux systèmes juridiques du Canada ${ }^{7} . »$

Bien que l'intention des instigateurs de la méthode de corédaction des lois fédérales fût noble - elle reposait sur une double visée: amélioration de la qualité des traductions et plus grand respect de la langue française -, il n'en demeure pas moins qu'elle aboutit à un rejet de la pratique de l'activité traduisante. Ce rejet, doublé des nombreux commentaires défavorables dont la traduction fut l'objet, ont mené à une forme de dévalorisation de la profession même de traducteur.

\section{Le traducteur juridique: une reconnaissance de plein droit?}

Il était assuré que le traducteur ou la traductrice juridique n’allait pas sortir indemne des nombreuses attaques faites à la traduction, ou encore d'un changement radical dans la façon de réaliser le bilinguisme législatif.

En 1969, Sussmann considère que le traducteur juridique n'est pas sûr de lui, qu'il n'a pas confiance en ses moyens, c'est pourquoi il produit une version littérale du texte original: "To return to the pitfalls of translating into French a statute drafted in English, we may note generally that very often the insecure translator, no doubt for safety's sake, has clung to too literal a translation of the original version » (p. 19, nos italiques). Premier conseiller législatif au ministère de la Justice du Canada, Lionel Levert note, dans un article paru dans la Revue de la common law en français en 2000, que cette insécurité du traducteur s'explique par un manque de connaissances juridiques: «Jusque dans les années 1970, les lois étaient rédigées en anglais, puis traduites en français par des traducteurs auxquels on ne reconnaissait aucune compétence particulière en droit et qui, de ce fait, étaient le plus souvent astreints à rendre servilement le message du texte anglais» (p. 129). Ainsi, ce n'est pas tant le procédé de traduction en lui-même qui est déficient, mais bien plutôt la formation des traducteurs qui est inadéquate, ce qui a pour conséquence immédiate de diminuer la qualité des traductions. À la fin des années 1980, les traducteurs n’avaient pas la double formation selon Roda P. Roberts: «[...] almost all professionals presently working in legal translation have formal training only in one of these two disciplines: some hold a degree in law (those working in the legal sections of the Translation Bureau of the Department of the Secretary of State, for example); others hold a degree in translation (translators working in Ontario's Legislative Counsel Office, for instance)» (1987: 8). De tels constats expliquent non seulement l'état des choses en matière de traduction juridique, mais sont révélateurs d'une non-reconnaissance de la profession même de traducteur.

D'autre part, cette mauvaise qualité des traductions réside peut-être aussi dans les conditions de travail des traducteurs, et non dans la nature de leur travail. En 1971, Claude-Armand Sheppard signait un rapport commandé par la Commission royale 
d'enquête sur le bilinguisme et le biculturalisme dans lequel il admettait que le Bureau de la traduction ne consacrait pas suffisamment de temps à la traduction des lois: "The Bureau is not a part of the Department of Justice and receives many assignments from other government departments. It cannot devote to the translation of statutes enough time for this arduous task» (p. 114). Ethel Groffier fait un constat similaire: «[...] les lois fédérales étaient — autrefois sans exception, aujourd'hui encore en majorité — rédigées en anglais, puis traduites, souvent dans des conditions de grande précipitation» (1989: 221).

Autre reproche souvent adressé au traducteur: le rôle de législateur qu'il s'accorde illégalement. Certains auteurs ont le regret de constater que le traducteur participe à la production du sens, qu'il crée le droit, au même titre que le législateur. Selon Pigeon, une telle mission ne relève ni des fonctions du légiste, ni de celles du traducteur. Or, tout en admettant le caractère critique de la traduction, l'ancien juge s'élève contre son pouvoir créateur:

Rien n'est plus difficile que de déceler [dans un texte de loi] les ambiguïtés insoupçonnées. À cet égard, la traduction dans l'autre langue est extrêmement utile parce que celui qui regarde le texte de façon à l'exprimer dans une autre langue est beaucoup plus porté que l'auteur à percevoir ce genre d'ambiguïtés. C'est pourquoi, dans une bonne rédaction législative, il faut que le traducteur ait accès au rédacteur de façon à pouvoir lui signaler toutes les ambiguïtés que le texte peut receler car, ces ambiguïtés, elles doivent disparaître. Si l'on ne se préoccupe pas de les faire disparaître par l'œuvre du rédacteur, ce qui arrive c'est que souvent c'est le traducteur qui les élimine en optant pour l'un ou l'autre des deux sens possibles. Alors il fait œuvre de législateur, et c'est ce qu'il ne doit pas faire. Il est arrivé assez souvent que les tribunaux aient interprété un texte voté par le Parlement canadien en adoptant le sens que révélait le texte français. Cependant, on sait qu'à Ottawa le traducteur n'a pas accès au rédacteur. Par conséquent, le texte français est présentement l'œuvre du traducteur seul. Chaque fois que ce texte a résolu une ambiguïté qui existait dans le texte anglais, c'est le traducteur qui a légiféré à l'insu du rédacteur. (1982: 81-82)

Pigeon est parfaitement conscient des mécanismes de la traduction, c'est-à-dire du rôle fondamental assumé par le traducteur dans la création du message d'arrivée, sur l'influence subjective exercée sur cette création. Il serait d'accord avec Folkart, qui écrit que: "Aucune citation, aucune paraphrase, aucune traduction, en effet, n'est jamais innocente. On cite, paraphrase, traduit toujours par intérêt. De manière encore plus essentielle, on cite, paraphrase, traduit toujours à partir d'une certaine position dans l'espace socioculturel, temporel, géographique. On ne saurait de ce fait ré-énoncer sans y mettre du sien» (1991: 14). Voilà précisément ce qui gêne Pigeon, qui ne reconnaît pas au traducteur de compétences interprétatives. Selon lui, ces compétences relèvent uniquement du rédacteur de la loi. On retrouve une position identique chez Covacs: «[...] la tradition a fait que, jusqu'à une date récente, les projets ont toujours été rédigés en anglais, puis simplement traduits en français. Ainsi, comme l'a fait remarquer un ancien juge de la Cour suprême du Canada [i.e. Pigeon], le traducteur se trouvait être abusivement le premier, et dans de nombreux cas le seul, interprète de la loi » (1982: 85). On pourrait bien sûr faire objection à de tels propos en posant la question de savoir comment on peut traduire un texte sans l'interpréter. D'autant plus que, au moment où il a publié ces lignes, Covacs était luimême traducteur. En réalité, Covacs déplore surtout le fait que, n’ayant pas de formation en droit, le traducteur n'aurait pas dû assumer la tâche du juriste. Enfin, 
Didier considère, lui aussi, que l'interprétation du texte de loi devrait relever uniquement du juriste: «Le langage du droit est [...] un domaine privilégié, à cause du petit nombre de connotations qui s'y trouvent. On y trouve cependant des «termes flous» du genre des «bonnes mœurs», du «bon père de famille» ou du «reasonable man». Mais ces mots n'ont pas de sens par eux-mêmes, ils n'en ont que par référence à un bagage culturel et juridique qui est connu du spécialiste. Ce sont des termes vides que l'on peut remplir comme on veut. Ce travail de remplissage n'est pas celui du traducteur, mais celui de l'interprète du droit» (1990: 222). Une fois encore, la fonction créatrice de la traduction est critiquée, le traducteur ne devant pas outrepasser sa mission. Ce dernier est donc perçu comme un être passif et transparent; il devient, dans les termes de Kasirer:

[...] a non-actor for law, whose task is limited to transcribing a legal text from one language to another without participating in the production of new ideas. The translator is often depicted, if at all, as a faceless player in the transmission of legal ideas and his or her work is not understood to be a communicative act distinct from that of the author of the source legal text. Plainly labouring under the weight of the dominant positivist ethic for interpretation in law, the translator is not imagined as a legitimate creative actor but as a simple mediator of legal ideas. (2001:333)

Folkart ne dit pas autre chose: «[...] la fiction du traducteur transparent est presque une convention de réception. Une première spécificité de la traduction réside donc dans ce qu'on pourrait appeler sa visée de transparence. [...] la traduction prétend ne point annexer ce discours qu'elle annexe pourtant en le filtrant à travers la subjectivité ré-énonciatrice et en le remédiatisant à travers la langue, l'hypersystème et le système culturel d'arrivée» $(1991$ : 218). Transparent et doué de la plus grande humilité, le traducteur a, de tout temps, été contraint (s'est-il contraint lui-même, parfois?) de s'effacer derrière l'auteur et le texte source. Le droit ne semble pas faire exception à la règle: le sujet traduisant doit s'abstenir de toute activité interprétative.

Il n'est donc pas étonnant, devant un tel portrait, qu'on ait encouragé la corédaction. Partant, une fois ce mode instauré, le travail du rédacteur est mis en valeur, tandis que celui du traducteur est discrédité. C'est, à tout le moins, ce que semble affirmer Covacs: «Du coup se trouve revalorisée la fonction du juriste francophone, passé de la situation de contre-réviseur à celle de rédacteur légiste... de plein droit » (1982: 86).

\section{Conclusion}

Bien qu'elles aient certes été justifiées, les critiques attaquant le littéralisme des traductions des lois fédérales ont jeté le discrédit sur l'ensemble de cette activité. En plus d'être le reflet de la stigmatisation générale que subit la traduction, la mauvaise presse dont sont l'objet cette profession et le produit qu'elle génère (le texte traduit) n'est sûrement pas étrangère au discours véhiculé par de nombreux juristes (Côté, Didier, Beaupré, Macdonald) pour qui traduire est soit un acte simple qui ne requiert aucune créativité, soit une opération qui a pour principal effet de dénaturer l'expression du droit.

Devant cet état de choses, on peut se demander s'il n'y a pas une légère contradiction dans les termes au sein du discours sur la traduction juridique. En effet, d'un côté, les auteurs postulent l'égale autorité des deux versions et l'outil critique ou 
interprétatif que constitue la traduction d'un texte législatif; et, de l'autre, ces mêmes auteurs accusent la traduction juridique d'être de mauvaise qualité et mettent de l'avant un moyen de la contourner comme la corédaction.

En fait, il s'agit d'une fausse contradiction. La prétendue mauvaise qualité des traductions n'élimine pas du même coup le caractère foncièrement herméneutique de cette activité. D'une part, les erreurs apparaissant dans une traduction peuvent servir à attirer l'attention sur une ambiguïté du texte original, sur un passage flou ou mal dit. Et, d'autre part, même si la traduction accuse des écarts flagrants, des interférences évidentes avec le texte original, il n'en demeure pas moins qu'elle reste un excellent moyen d'avoir accès au sens de ce texte, puisque le traducteur représente le premier lecteur, par conséquent le premier interprète de la loi.

La corédaction demeure bien sûr un moyen efficace d'exprimer le droit dans les deux langues officielles. Mais la traduction pourrait assumer le même rôle si les modalités de sa pratique étaient changées, car derrière l'apparente contradiction qui frappe le discours juridique se profile une évaluation de ce qu'on considère être une bonne et une mauvaise traduction. Le droit fait ressortir avec acuité la nécessité d'opter, non pas pour une traduction opaque, calquant l'original, mais bien plutôt pour une traduction adaptée et libre qui pourrait conduire à une véritable interprétation du texte original. À partir du moment où les critères seraient changés, on pourrait avoir recours à la traduction, et sa fonction herméneutique serait ainsi pleinement mise à profit.

\section{NOTES}

1. L'auteure tient à remercier Hélène Buzelin, Jeanne Dancette et Nicholas Kasirer de leur relecture attentive du présent article, de leurs commentaires pertinents et de leurs précieux conseils. Elle souhaite également exprimer sa reconnaissance à son assistante de recherche, Cinzia Carlone, étudiante au Département de linguistique et de traduction de l'Université de Montréal, qui a assumé avec rigueur, efficacité et patience la collecte des données pour cette recherche. L'auteure remercie également de son soutien financier le Conseil de recherches en sciences humaines du Canada (CRSH).

2. Voir C.P.R. c. Robinson [1891] 19 S.C.R. 292, mod. par [1892] A.C. 481 C. privé et The King c. Dubois [1935] S.C.R. 378 cités respectivement par Beaupré, 1986, p. 20, n. 28 et p. 7, n. 12. Beaupré souligne par ailleurs le fait que l'article 133 de la Loi constitutionnelle de 1867 ne postule pas explicitement l'égale autorité des deux versions, mais que les tribunaux en ont fait une interprétation qu'il qualifie de «créative» (1986, p. 7). L'article 8, premier alinéa, de la Loi sur les langues officielles (1969) postule explicitement l'égalité des deux versions: «Dans l'interprétation d'un texte législatif, les versions des deux langues officielles font pareillement autorité». Depuis 1969, la loi a été abrogée et remplacée en 1988 par une nouvelle Loi sur les langues officielles qui ne comporte plus de dispositions sur l'interprétation des textes bilingues (Côté, 1999, p. 410). Toutefois, l'article 18(1) de la Loi constitutionnelle de 1982, dispose que: "Les lois, les archives, les comptes rendus et les procès-verbaux du Parlement sont imprimés et publiés en français et en anglais, les deux versions des lois ayant également force de loi et celles des autres documents ayant même valeur» (dans Labelle, 2000, p. 271 et Levert, 2000, p. 128).

3. Dans son article «What is vie commune? Qu'est-ce que living together?», Kasirer explore la distance et les rapprochements existant entre deux versions d'une même disposition du Code civil du Québec et montre à quel point le sens n'est pas fixé dans l'une ou l'autre version, mais bien plutôt, « [...] floating somewhere between them» (1997, p. 532). On lira également, sur le caractère bilingue du Code, l'avant-propos de l'édition critique originale (1997) par Brisson et Kasirer.

4. J.A. Clarence Smith (1983, p. 603) fournit l'exemple tiré du Code criminel (L.R.C. 1970, chap. C-34, art. 228).

5. Tiré de la Water Carriage of Goods Act (R.S.C. 1952, c. 291, Art. IV, s. 2 (p)), cité par Honsberger (1965, p. 323). 
6. Sauf pour Didier, qui déclare sans détour que: «L'objectif des nouvelles techniques [de rédaction, comme la corédaction] est d'éliminer à terme la traduction législative» (1990, p. 322).

7. Commissaire aux langues officielles, Sixième Rapport Annuel, Ottawa, ministre des Approvisionnements et Services Canada, 1977, p. 151, cité par Beaupré, 1986, p. 212.

\section{RÉFÉRENCES}

Beaupré, R. M. (1986): Interprétation de la législation bilingue. Traduit de l'anglais par Ethel Groffier-Atala, Montréal, Wilson \& Lafleur.

Bélair, N. (1987): "Législation bilingue - la production de la seconde version ». L'Actualité terminologique/Terminology Update, 20, 6, p. 6-8.

Berman, A. (1995): Pour une critique des traductions: John Donne. Paris, Gallimard.

Bertrand, G. (1987): "Codification, révision et rédaction des lois en régime fédéral de droit jurisprudentiel anglais et en situation de bilinguisme officiel français-anglais: l'expérience canadienne ». L'Actualité terminologique/Terminology Update, 20, 6, p. 4-5.

Brisset, A. (1990): Sociocritique de la traduction. Théâtre et altérité au Québec (1968-1988). Longueuil, Le Préambule, (Coll. «L'Univers des discours»).

Côté, P.-A. (1999): Interprétation des lois. $3^{\text {e }}$ édition, Montréal, Thémis.

Covacs, A. (1982) : «La réalisation de la version française des lois fédérales du Canada ». Langage $d u$ droit et traduction, sous la direction de Jean-Claude Gémar. Québec, Linguatech, p. 83-100.

Didier, E. (1990): Langues et langages du droit. Étude comparative des modes d'expression de la Common Law et du Droit Civil, en français et en anglais. Montréal, Wilson \& Lafleur.

Folkart, B. (1991): Le conflit des énonciations. Traduction et discours rapporté. Candiac, Balzac, coll. «L'Univers des discours».

Gémar, J.-C. (1995): Traduire ou l'art d'interpréter. Fonctions, statut et esthétique de la traduction. Tome 1 : Principes. Sainte-Foy (Québec), Presses de l'Université du Québec, 257 p.

Groffier, E. (1989): «Le juge Pigeon et la langue du droit». Mélanges Louis-Philippe Pigeon, Montréal, Wilson \& Lafleur, p. 215-234.

Honsberger, J. D. (1965) : «Bilingualism in Canadian Statutes ». Revue du barreau canadien, 43, p. 314-336.

Kasirer, N. (2001): "François Gény's libre recherche scientifique as a Guide for Legal Translation ». Louisiana Law Review, Winter, 61, 2, p. 331-352.

— (1994) : «Dire ou définir le droit?»: Thémis, 28, 1, p. 143-173.

Levert, L. A. (2000): "La cohabitation du bilinguisme et du bijuridisme dans la législation fédérale canadienne: mythe ou réalité?» Revue de la common law en français, 3, p. 127-136.

Macdonald, R. A. (1997): «Legal Bilingualism». Revue de droit de McGill, 42, p. 119-168.

Pigeon, L.-P. ([1965], 1982) : Rédaction et interprétation des lois. Québec, Éditeur officiel.

Roberts, R. P. (1987) : "Legal Translator and Legal Interpreter Training in Canada». L'Actualité terminologique/Terminology Update, 20, 6, p. 8-10.

Sheppard, C.-A. (1971): The Law of Languages in Canada. Ottawa, Information Canada, «Studies of the Royal Commission on Bilinguism and Biculturalism», 10.

Sмith, E. (1979): «Peut-on faire de la common law en français?» La revue de l'Université de Moncton, 12, 2-3, septembre-décembre, p. 39-46.

Smith, J.A.C. (1983) : «La common law en français». The Canadian Bar Review, 61, p. 595-608.

Sussmann, F. B. (1969): «Bilingualism and the Law in Canada».Travaux du sixième colloque international de droit comparé. Ottawa, Éditions de l'Université d'Ottawa, p. 9-38. 